\title{
Sistema de evaluación en las prácticas clínicas de la titulación de Medicina
}

\author{
Carmen I. Reyes-García, Fátima Sosa-Moreno, Rosa Marchena-Gómez, Joaquín Marchena-Gómez
}

Resumen. Este artículo describe un proyecto de innovación educativa realizado por un grupo interdisciplinar de profesores de la Universidad de Las Palmas de Gran Canaria. Su objetivo ha sido realizar un diagnóstico de la evaluación de las prácticas que se están desarrollando en la titulación de Medicina. Los procedimientos metodológicos se han basado en la cumplimentación de un cuestionario por parte del profesorado y en el análisis documental de los proyectos docentes. Después de analizar los resultados, hemos constatado que el sistema de evaluación de las prácticas clínicas que se está desarrollando actualmente en nuestra universidad no se alinea con el modelo actual demandado desde el Espacio Europeo de Educación Superior.

Palabras clave. Evaluación auténtica. Evaluación de competencias. Proyectos docentes.

\section{Assessment system for clinical practices in the qualifications of Medicine}

Summary. This article refers to an educational innovation project carried out by an interdiciplinary group of lecturers from the University of Las Palmas de Gran Canaria. Their main target has been to analyze the practice assessment in the qualifications of Medicine in order to diagnose it. The procedures for the collection of information have been based on the filling in of a questionnaire addressed to qualified lecturers and on the documentary analysis of the teaching projects. In general, once having analyzed the results, we have verified that the assessment system for clinical practices, actually being developed at our university isn't in line with the present model demanded by the European Higher Education Area.

Key words. Assessment of competencies. Authentic assessment. Teaching programmes.

\section{Introducción}

Asumiendo el enfoque de lo que debe considerarse una evaluación auténtica, mantenemos que son necesarias algunas propuestas de mejora en las asignaturas del nuevo Grado de Medicina para que estén acordes con las demandas del Espacio Europeo de Educación Superior (EEES) y con la nueva forma de entender la evaluación. Desde esta perspectiva, Epstein [1] ofrece una clasificación actualizada de los procedimientos de evaluación potencialmente útiles en medicina donde incluye no sólo los más utilizados, sino también otros que emergen en la actualidad; aporta las ventajas y limitaciones de cada uno e identifica algunos retos en la evaluación de las competencias profesionales de esta titulación. En nuestro país, la agencia catalana para la calidad del sistema universitario (AQU Catalunya) [2] también ha elaborado un informe exhaustivo acerca de la evaluación de las competencias en medici- na, en el que se detalla una amplia clasificación de procedimientos de evaluación.

Desde nuestro grupo de investigación hemos constituido un proyecto de innovación educativa interdisciplinar con el propósito de diagnosticar la evaluación de las prácticas de las asignaturas clínicas en la titulación de Medicina, ya que sólo así serán sólidas las propuestas de mejora que podamos establecer. El hecho de centrarnos en el ámbito de las prácticas responde fundamentalmente a que es en el desarrollo de este aprendizaje donde el alumno tiene que aplicar un mayor número de competencias profesionales y no tanto de conocimiento, situación que genera, por tanto, mayor complejidad para que el profesor las evalúe.

Con este amplio propósito, nuestro trabajo se ha marcado los siguientes objetivos:

- Describir el sistema de evaluación de prácticas clínicas analizando y contrastando lo que opinan los profesores de sus propias prácticas evaluati-
Departamento de Educación; Facultad de Formación del Profesorado (C.I. Reyes-García, F. Sosa-Moreno, R. MarchenaGómez). Departamento de Ciencias Médicas y Quirúrgicas; Facultad de Ciencias de la Salud (J. Marchena-Gómez). Universidad de Las Palmas de Gran Canaria. Las Palmas de Gran Canaria, España.

Correspondencia:

Dra. Carmen Isabel Reyes García. Departamento de Educación. Facultad de Formación del Profesorado. Universidad de Las Palmas de Gran Canaria. Santa Juana de Arco, 1. E-35004 Las Palmas de Gran Canaria.

E-mail:

creyes@dedu.ulpgc.es

Conflicto de intereses: No declarado.

Conflict of interests: None declared.

(c) 2013 FEM 
vas y lo que se plasma en los proyectos docentes de las asignaturas (plan de estudios de 1997, BOE n. ${ }^{\circ}$ 176, de 23 de julio de 1997).

- Detectar la consistencia o validez del sistema de evaluación de las diferentes asignaturas a partir de los hallazgos obtenidos

- Valorar la distancia o discrepancia entre la evaluación detectada y el nuevo modelo de evaluación que se requiere de acuerdo a las exigencias del EEES.

\section{Materiales y métodos}

Situamos nuestro trabajo bajo el enfoque de una investigación cualitativa. La muestra seleccionada se acogió a la selección de un caso (la Facultad de Medicina de la Universidad de Las Palmas de Gran Canaria) siguiendo un muestro intencional. Con el mismo tipo de muestreo, pero ya con un criterio teórico, seleccionamos varios proyectos docentes pertenecientes al plan de estudios de 1997, que correspondían a aquellas asignaturas que se encargaban del estudio de las enfermedades del paciente y que incorporaban diferenciadamente contenidos teóricos y prácticas clínicas. Se incluyó asimismo la asignatura de 'Prácticas clínicas'.

Los instrumentos utilizados fueron un cuestionario y el análisis de documentos. El cuestionario recogió la opinión de los profesores sobre la evaluación de las prácticas clínicas que estaban realizando. Tras un proceso de validación fue contestado por 31 docentes. Los resultados, convertidos en datos cuantitativos, se analizaron mediante técnicas estadísticas descriptivas a través del programa SPSS v. 18.

El análisis de documentos se aplicó a 20 proyectos docentes de materias clínicas. Los hallazgos de los proyectos se localizaron siguiendo procedimientos de investigación derivados del análisis de contenido [3], por lo que se estableció un sistema categorial que clasificó la información (Tabla I).

\section{Resultados}

Hemos hallado que las prácticas se diseñan de manera heterogénea en función de la asignatura que se trate. No todos los docentes planifican este ámbito incorporando los elementos curriculares básicos.

Independientemente del diseño de este elemento curricular, el profesorado encuestado ha exteriorizado una serie de problemas o necesidades con respecto a la evaluación de las prácticas. El 37\% del profesorado consideraba que era necesario un número mayor de profesores, y el $28 \%$, que el tiempo dedicado a las prácticas debía ampliarse y el número de alumnos por profesor debía reducirse. También, aunque en pequeños porcentajes, aparecieron señalados otros problemas relacionados con la información al alumnado, la evaluación, la falta de coordinación y la falta de motivación del profesorado.

La necesaria coordinación entre los tutores no existía en la opinión de alrededor de las tres cuartas partes del profesorado encuestado.

Atendiendo a la categorización del análisis efectuado, en las tablas I y II se detallan los resultados arrojados desde el análisis de los proyectos docentes y el cuestionario.

\section{Discusión}

A partir de los resultados expuestos, y teniendo en cuenta los objetivos que se habían fijado en este trabajo, planteamos las siguientes consideraciones:

\section{Sistema de evaluación en las prácticas clínicas}

Se trata de un modelo tradicional centrado en la evaluación de resultados y en el que se utiliza, mayoritariamente, el examen y la asistencia como instrumentos de evaluación, aunque en ciertos casos aparecen instrumentos alternativos.

Estamos ante una evaluación que tiene una función burocrática, de certificación. En nuestra universidad, las prácticas clínicas reciben menos atención a la hora de ser planificadas que los conocimientos teóricos de la materia, puesto que los profesores diseñan sólo alguno o algunos de sus elementos; incluso hemos hallado algunos programas que no recogen ninguna referencia a la evaluación de las prácticas. Por ello, al igual que ocurre en otras muchas facultades de medicina, existe una gran necesidad de mejorar la evaluación de competencias [1], en especial de las referidas a las actividades prácticas [4].

Buena parte del profesorado es consciente de esta problemática. Los profesores saben que la evaluación de las prácticas no es adecuada, que el alumnado recibe poca información sobre los elementos de la evaluación, que la coordinación entre los tutores es escasa y que el alumnado tampoco recibe la información adecuada a lo largo del desarrollo de las prácticas.

Se detecta igualmente confusión entre el profesorado respecto a los términos referidos a la evaluación. Se aportan distintos significados a un mismo elemento de la evaluación, o bien se confunden 
Tabla I. Elementos de la evaluación recogidos en los programas docentes de las asignaturas clínicas de medicina.

\begin{tabular}{|c|c|c|c|c|c|c|c|c|c|c|c|}
\hline & \multirow{3}{*}{$\begin{array}{l}\text { Objetos de } \\
\text { evaluación }\end{array}$} & \multicolumn{4}{|c|}{$\begin{array}{l}\text { Criterios de } \\
\text { evaluación }\end{array}$} & \multirow{3}{*}{ Procedimiento } & \multicolumn{2}{|c|}{$\begin{array}{l}\text { Criterios de } \\
\text { calificación o corrección }\end{array}$} & \multirow{3}{*}{ Momentos } & \multirow{3}{*}{ Agentes } & \multirow{3}{*}{ Adaptaciones } \\
\hline & & \multicolumn{2}{|c|}{ Explícitos } & \multicolumn{2}{|c|}{ Implícitos } & & \multirow{2}{*}{$\begin{array}{l}\text { Relativos } \\
\text { a asistencia }\end{array}$} & \multirow{2}{*}{$\begin{array}{l}\text { Relativos } \\
\text { a instrumentos } \\
\text { de evaluación }\end{array}$} & & & \\
\hline & & Inc. & Comp. & Inc. & Comp. & & & & & & \\
\hline Patología general & $x$ & & & & & Examen & & & & $x$ & \\
\hline Fundamentos de cirugía & $x$ & & & $x$ & & & & & & $x$ & \\
\hline $\begin{array}{l}\text { Enfermedades de los aparatos } \\
\text { circulatorio y respiratorio }\end{array}$ & & $\times$ & & & & & $\times$ & & & $\times$ & \\
\hline $\begin{array}{l}\text { Enfermedades del aparato } \\
\text { digestivo, del sistema endocrino, } \\
\text { del metabolismo y la nutrición }\end{array}$ & & $\times$ & & & & $\begin{array}{l}\text { Examen, } \\
\text { libreta de prácticas }\end{array}$ & & & & & \\
\hline $\begin{array}{l}\text { Enfermedades del sistema } \\
\text { inmune, de la sangre y de } \\
\text { los órganos hematopoyéticos }\end{array}$ & $x$ & & & & $\times$ & & $x$ & & & & \\
\hline Enfermedades del sistema nervioso & $\times$ & $\times$ & & & & & & & & & \\
\hline Oftalmología & & & & $x$ & & & $\times$ & & & $x$ & \\
\hline Obstetricia y ginecología & & $x$ & & & & $\begin{array}{l}\text { Examen, } \\
\text { libro del alumno }\end{array}$ & $x$ & & & $x$ & \\
\hline Estomatología médico-quirúrgica & & $\times$ & & & & & $x$ & & & & \\
\hline $\begin{array}{l}\text { Enfermedades infecciosas } \\
\text { y toxicología médica }\end{array}$ & $x$ & & & & & Examen & $x$ & $\times$ & & & \\
\hline $\begin{array}{l}\text { Enfermedades del riñón } \\
\text { y de vías urinarias }\end{array}$ & & & & & $x$ & 'Folio reflexivo' & $x$ & & & $x$ & \\
\hline $\begin{array}{l}\text { Enfermedades del } \\
\text { aparato locomotor }\end{array}$ & & $x$ & & & & $\begin{array}{l}\text { Cuaderno de } \\
\text { prácticas (diario) }\end{array}$ & $x$ & & & & \\
\hline Otorrinolaringología & $\times$ & & & & & $\begin{array}{l}\text { servación sistemática, } \\
\text { memoria, ficha }\end{array}$ & & & & & \\
\hline Dermatología y venereología & $\times$ & $\times$ & & & & Examen & & $\times$ & & & \\
\hline Psiquiatría & & $x$ & & & & Memoria & $x$ & & & & \\
\hline Pediatría & & $x$ & & & $x$ & $\begin{array}{l}\text { Examen, } \\
\text { ficha práctica }\end{array}$ & & & & & \\
\hline Geriatría & $\times$ & & & & $x$ & Examen & & & & & \\
\hline Cancerología general & $x$ & $x$ & & & & $\begin{array}{c}\text { Examen } \\
\text { (oral-escrito) }\end{array}$ & & & & & \\
\hline Práctica clínica & & $x$ & & & & Examen & & $x$ & & $x$ & \\
\hline Medicina de urgencias & $x$ & & & & & & & & & & \\
\hline
\end{tabular}


Tabla II. Frecuencias de la preguntas del cuestionario cumplimentado por el profesorado.

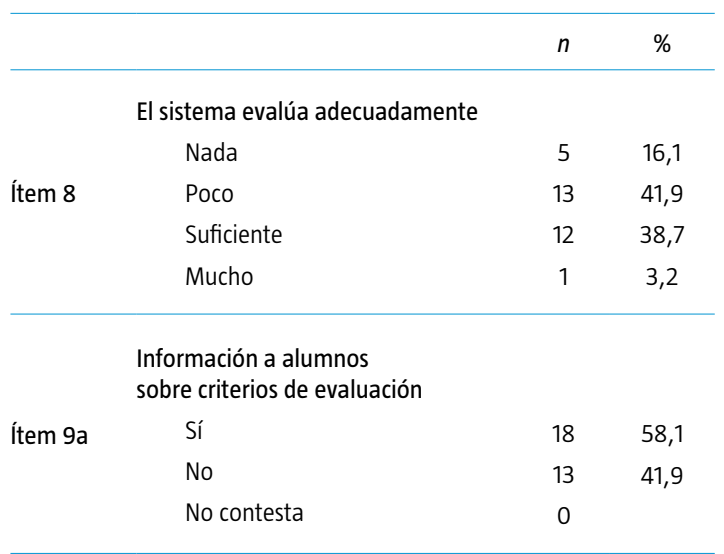

\begin{tabular}{|c|c|c|c|}
\hline \multirow{4}{*}{ Ítem 9b } & $\begin{array}{l}\text { Información a alumnos sobre } \\
\text { procedimientos de evaluación }\end{array}$ & & \\
\hline & Sí & 16 & 53,3 \\
\hline & No & 14 & 46,7 \\
\hline & No contesta & 1 & \\
\hline
\end{tabular}

\begin{tabular}{|c|c|c|c|}
\hline \multirow{4}{*}{ Ítem 9c } & $\begin{array}{l}\text { formación a alumnos } \\
\text { bre criterios de calificación }\end{array}$ & & \\
\hline & Sí & 17 & 56,7 \\
\hline & No & 13 & 43,3 \\
\hline & No contesta & 1 & \\
\hline
\end{tabular}

\begin{tabular}{|c|c|c|c|}
\hline \multirow{4}{*}{ Ítem 9d } & $\begin{array}{l}\text { formación a alt } \\
\text { bre momento }\end{array}$ & & \\
\hline & Sí & 13 & 43,3 \\
\hline & No & 17 & 56,7 \\
\hline & No contesta & 1 & \\
\hline
\end{tabular}

\begin{tabular}{|c|c|c|c|}
\hline \multicolumn{4}{|c|}{ Información durante las prácticas } \\
\hline & Nada & 6 & 19,4 \\
\hline \multirow[t]{3}{*}{ Ítem 10} & Poco & 12 & 38,7 \\
\hline & Suficiente & 13 & 41,9 \\
\hline & Mucho & 0 & \\
\hline
\end{tabular}

\begin{tabular}{|c|c|c|c|}
\hline \multirow{6}{*}{ Ítem 11} & \multicolumn{3}{|c|}{ Grado de coordinación de los tutores } \\
\hline & Nada & 10 & 34,5 \\
\hline & Poco & 11 & 37,9 \\
\hline & Suficiente & 8 & 27,6 \\
\hline & Mucho & 0 & \\
\hline & No contesta & 2 & \\
\hline
\end{tabular}

unos elementos con otros (criterios de evaluación, procedimientos, etc.). Esto provoca falta de comprensión y transparencia, e incluso podríamos decir que lesiona uno de los derechos fundamentales de los estudiantes: conocer previamente qué y cómo va a ser evaluado.

\section{Consistencia o validez del sistema de evaluación}

Existe una falta de validez del sistema de evaluación recogido en casi la totalidad de los proyectos docentes. Esto se debe a diversos factores que, en la mayor parte de los casos, se entremezclan. Algunos proyectos no especifican algunos de los elementos clave de la evaluación: criterios, procedimientos, etc. Esta omisión va en contra del derecho de los estudiantes a estar informados sobre el proceso de evaluación según las normativas prescritas al respecto.

En otros proyectos, el problema es más grave porque los elementos de la evaluación no están alineados, es decir, son incongruentes con la naturaleza y diversidad del objeto estudiado: por ejemplo, un tercio de los profesores opta por la asistencia; otros eligen el examen como único instrumento de evaluación, olvidando que las competencias clínicas son un 'saber actuar' complejo que implica la movilización de recursos sobre los que se apoya la competencia y, por eso, a la hora de evaluarlas, es necesario presentar a los estudiantes una serie de situaciones o procedimientos de evaluación que permitan documentar su trayectoria de desarrollo a lo largo de la formación y certificar al final el grado de desarrollo alcanzado. En definitiva, la evaluación de las prácticas clínicas deberá realizarse en situaciones lo más 'auténticas' posible, lo más próximas al contexto profesional, lo que permitirá realizar tareas completas, complejas y significativas [5].

En general, los profesores no utilizan pruebas variadas de desempeño, es decir, manifestaciones externas que evidencien el nivel de aprendizaje del estudiante alcanzado en las prácticas clínicas: conocimientos, habilidades y valores. Esta limitación en la evaluación de las prácticas clínicas, especialmente en las prácticas externas tuteladas, ha sido corroborada por recientes publicaciones españolas [5-8] y extranjeras [9].

\section{Distancia o discrepancia entre la evaluación detectada y el nuevo modelo de evaluación}

En el contexto que analizamos, hemos hallado que la evaluación 'auténtica' o del desempeño prácticamente no se ha utilizado porque no se cumplen sus características: tareas auténticas, criterios de evaluación relevantes, información individualizada, etc. Por ello pensamos, al igual que Nolla-Domenjó [10], que el mayor desafío en la evaluación de la educación médica reside en la valoración de com- 
petencias en entornos simulados y, más aún, en contextos reales, como sucede en las prácticas clínicas. Se precisa una evaluación del proceso, centrada en el aprendizaje de carácter colaborativo y con posibilidad de retroalimentación, que permita verificar el aprendizaje de los estudiantes en múltiples y variadas ocasiones utilizando memorias prácticas, informes, carpetas, la realización de actividades in situ, etc. Para aumentar la fiabilidad de estos procedimientos será indispensable estandarizar los criterios e indicadores de evaluación a través del consenso entre todos los agentes evaluadores. Para ello, convendría elaborar instrumentos de evaluación de las competencias que, como las rúbricas, sirvan para documentar la trayectoria de desarrollo a lo largo del proceso de formación y para valorar la calidad de las actuaciones de los estudiantes.

En definitiva, resulta manifiesta la falta de formación didáctica del profesorado con respecto a la evaluación de los aprendizajes. Hay que considerar que la concepción tradicional y predominante sobre la naturaleza y propósito de la evaluación que se detecta en ellos puede limitar el uso de modelos alternativos como la evaluación auténtica. Por tan- to, el profesorado necesita implicarse en un proceso que le permita integrar el nuevo modelo de evaluación en el currículo a través de las distintas fases de formación: la sensibilización, la conceptualización, la comprensión y el uso de sistemas de evaluación auténticos que impliquen a los estudiantes en sus procesos de diseño y que atiendan a la diversidad.

\section{Bibliografía}

1. Epstein R. Assessment in medical education. Eval Health Prof 2007; 27: 239-50.

2. Carreras-Barnés J. Guía para la evaluación de competencias en Medicina. Barcelona: AQU Catalunya; 2009.

3. Krippendorff K. Metodología de análisis de contenido. Teoría y práctica. Barcelona: Paidós; 1990.

4. Norcini JJ, McKinley DW. Assessment methods in medical education. Teach Educ 2007; 23: 239-50.

5. Arnalich F. Adaptación al nuevo grado de Medicina al Espacio Europeo de Educación Superior. ¿Cuál es la aportación de Bolonia? Educ Med 2010; 13 (Supl 1): S53-7.

6. Argudin Y. Educación basada en competencias. Nociones y antecedentes. México: Trillas; 2007.

7. Carretero J. Técnicas y recursos educativos en la evaluación de medicina. Educ Med 2010; 13 (Supl 1): S9-12.

8. Millán J. Evaluación del alumno. Educ Med 2010; 13 (Supl 1): S57-60.

9. Panzarella KJ, Manyon AT. A model for integrated assessment of clinical competence. J Allied Health 2007; 36: 157-64.

10. Nolla-Domenjó M. La evaluación en educación médica. Principios básicos. Educ Med 2009; 12: 223-9. 